\title{
Vol. 69, No. 21
}

In the report "Evaluation of a Program to Improve Linkage to and Retention in Care Among Refugees with Hepatitis B Virus Infection - Three U.S. Cities, 2006-2018," on page 650, Katherine Yun, Children's Hospital of Philadelphia should have been listed in the Acknowledgments section. 